\title{
Supplemental Information for
}

\section{Enhanced Plasma Generation from Metal Nanostructures}

\section{via Photoexcited Hot Electrons}

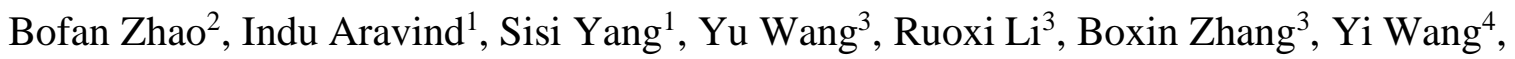
Jahan M. Dawlaty ${ }^{4}$, and Stephen B. Cronin ${ }^{* 1,2,4}$

${ }^{1}$ Department of Physics and Astronomy, ${ }^{2}$ Ming Hsieh Department of Electrical Engineering, ${ }^{3}$ Mork Family Department of Chemical Engineering and Materials Science

${ }^{4}$ Department of Chemistry

University of Southern California, Los Angeles, CA 90089, USA

*Corresponding author email: scronin@usc.edu 

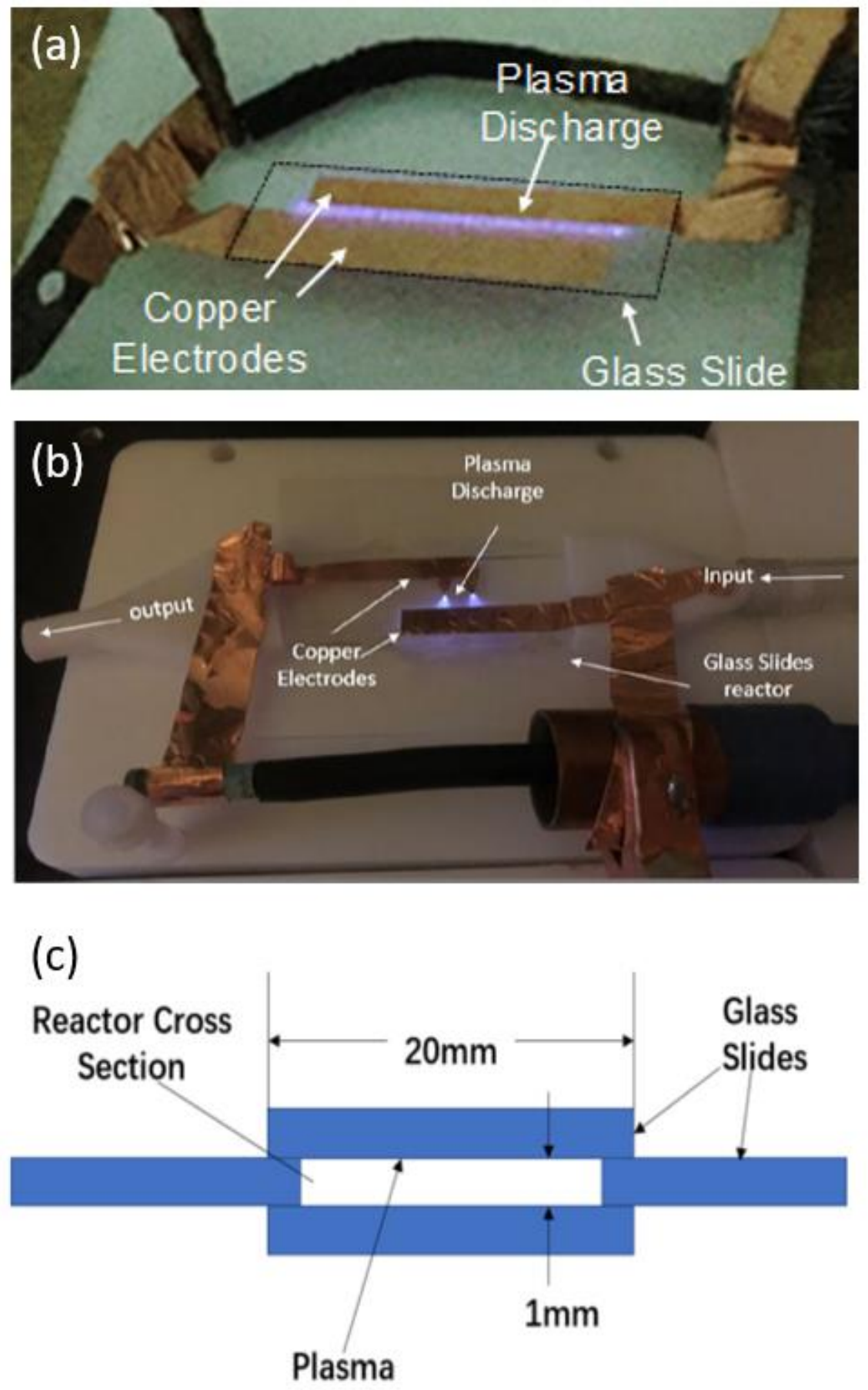

Figure S1. (a) Photograph of the nanosecond pulse plasma discharged across a $5 \mathrm{~mm}$ gap on a glass slide and (b) an image of the glass slide reactor together with (c) cross-sectional diagram. 
(a)

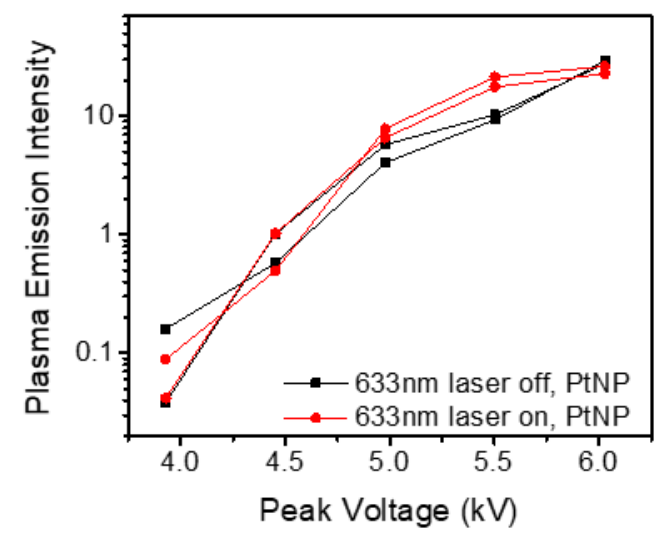

(b)

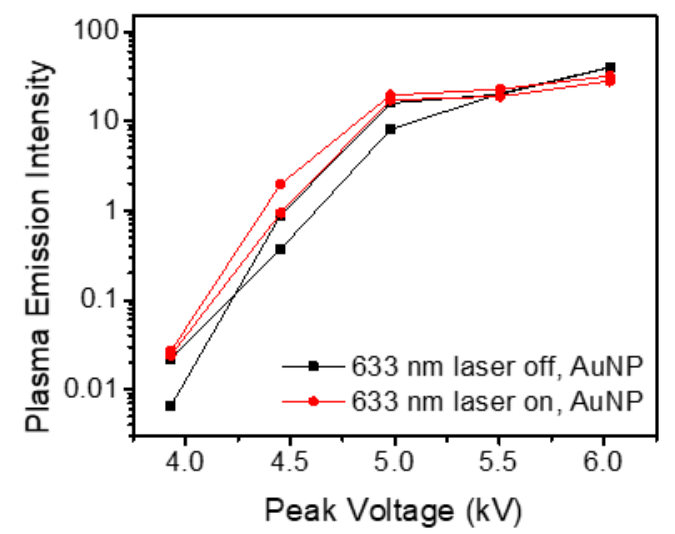

(c)

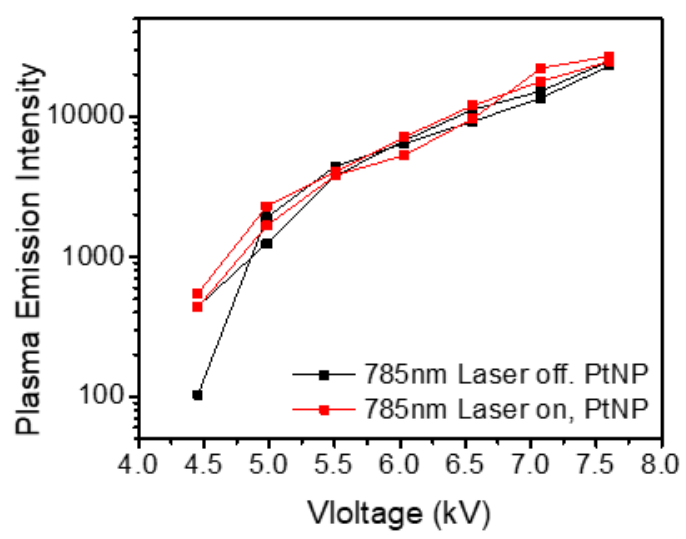

(d)

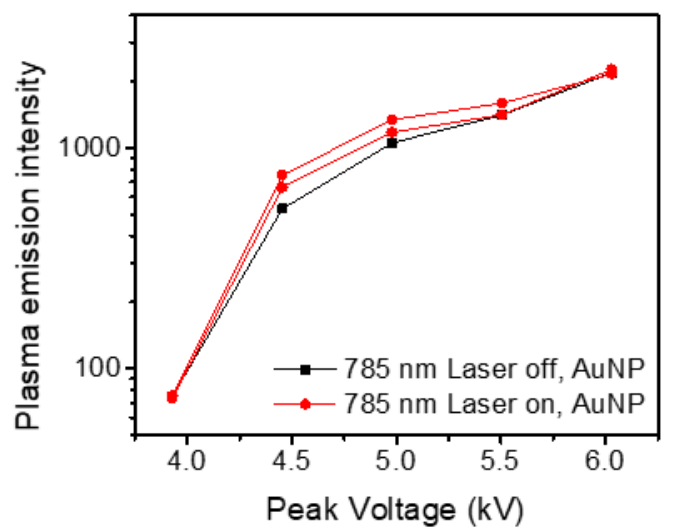

Figure S2. Plasma emission intensity of argon $912.3 \mathrm{~nm}$ emission peak discharged across (a) (c) Pt nanoparticles and (b) (d) Au nanoparticles with and without $633 \mathrm{~nm}$ or $785 \mathrm{~nm}$ laser irradiation. No obvious enhancement can be observed with laser irradiation of both wavelength for both metal nanoparticles. 
(a)

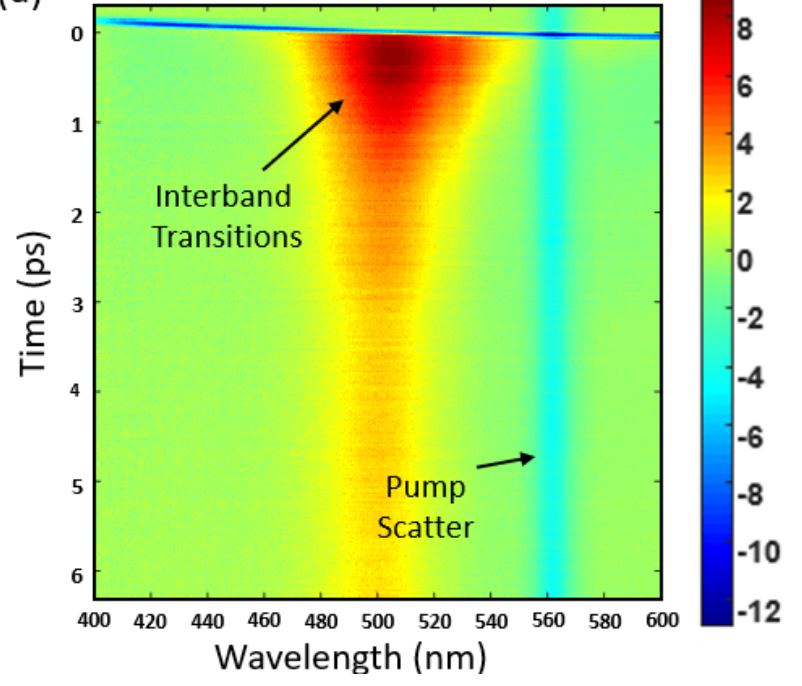

(b)

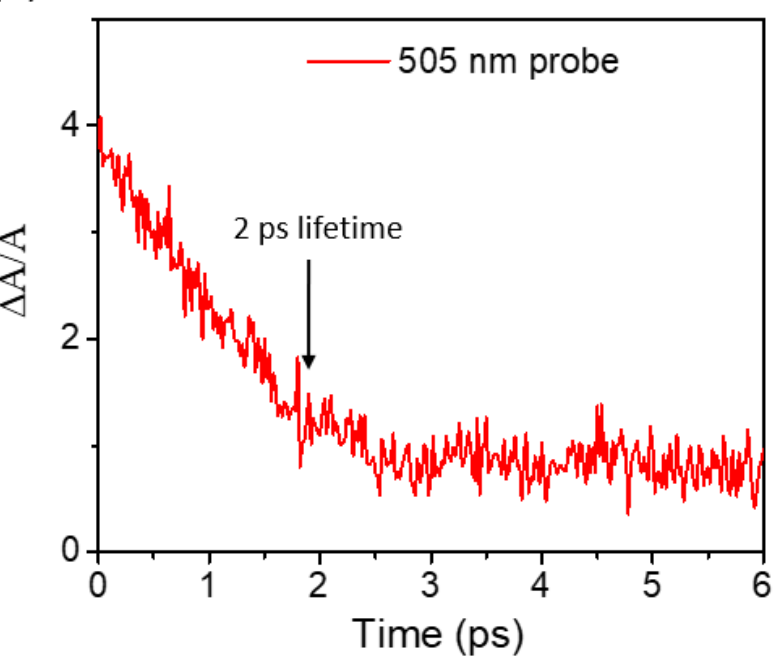

Figure S3. (a) Transient absorption spectra measured on a Au grating nanostructure plotted as a function of wavelength and time. (b) Time dependence obtained with a $505 \mathrm{~nm}$ probe wavelength. These results suggest that the lifetime of hot electrons inside gold nanostructures can be extend several psec. ${ }^{1}$

\section{Reference}

1. Wang, Y.; Shi, H.; Shen, L.; Wang, Y.; Cronin, S. B.; Dawlaty, J. M., Ultrafast Dynamics of Hot Electrons in Nanostructures: Distinguishing the Influence on Interband and Plasmon Resonances. ACS Photonics 2019, 6 (9), 2295-2302. 